\title{
Von Recklinghausen disease (neurofibromatosis type 1): beyond skin involvement
}

\author{
Sara M Rocha, Maria Betânia Ferreira, Rosa Ribeiro, João Correia
}

Department of Internal Medicine, Centro Hospitalar Porto, Porto, Portugal

\section{Correspondence to} Dr Sara M Rocha, saraisabelrocha@gmail.com

\section{DESCRIPTION}

A 35-year-old black woman with Von Recklinghausen disease (or neurofibromatosis type 1) presented multiple café-au-lait spots, axillary freckling and multiple cutaneous neurofibromas, which were more expressive in the abdomen and trunk (figure 1). In the course of the disease, she developed nodular and diffuse plexiform neurofibromas, associated with mild pain and skin disfigurement (figure 1D). A CT scan was performed when she complained of lumbar pain, although she had a normal neurological examination. This study showed a heterogeneous lumbar mass involving the neurological structures and the electrophysiological examination proved a decreased conduction velocity in the left lower limb, supporting the diagnosis of neuropathy.
According to her disease, this proximal nerve hypertrophy could have two major aetiologies-a benign internal complex plexiform neurofibroma or a malignant peripheral nerve sheath tumour (MPNST). Other genetic diseases, such as Charcot-Marie-Tooth disease, Dejerine-Sottas disease and Noonan disease, were considered and excluded considering the absence of personal and family history suggestive of those diseases. Acquired causes of neuropathy, like chronic inflammatory demyelinating polyradiculoneuropathy, were also ruled out after several studies (haemoglobin, erythrocyte sedimentation rate, fasting morning glucose levels, serum urea and creatinine, electrolytes, liver function, serum immunoelectrophoresis, serum immunophenotyping, antinuclear antibody, thyroid-stimulating

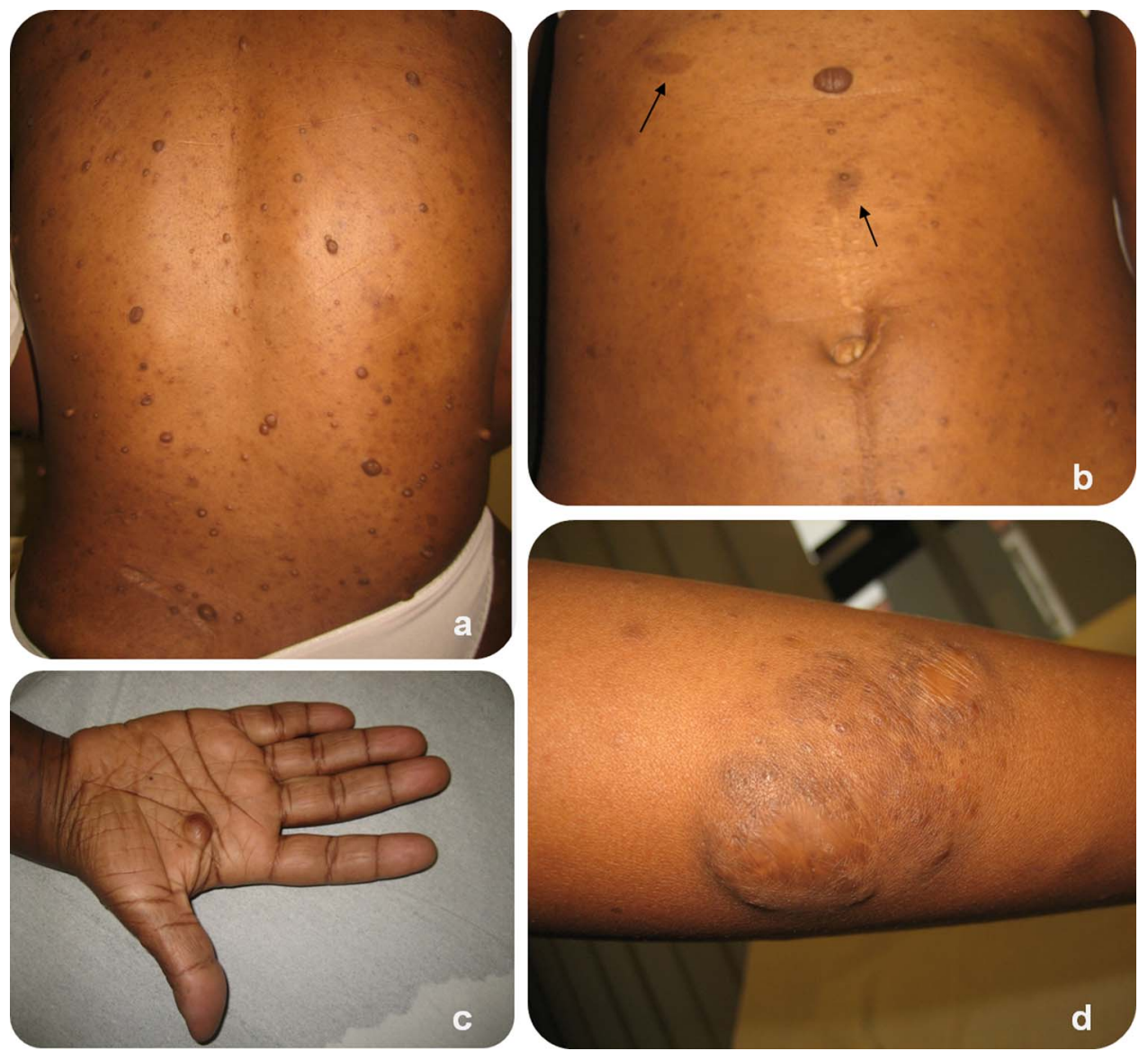

Figure 1 Patient photography's: (A) skin-coloured soft papules and nodules, consistent of multiple cutaneous neurofibromas in the back, which increased in size and number with age; (B) flat uniformly hyperpigmented macules known as "café-au-lait" spots are also visible in the abdomen; (C) a subcutaneous neurofibroma on the right hand, presented as a firm and tender nodule; (D) a diffuse plexiform neurofibroma on the right lower member representing an important cause of morbidity and disfigurement. 


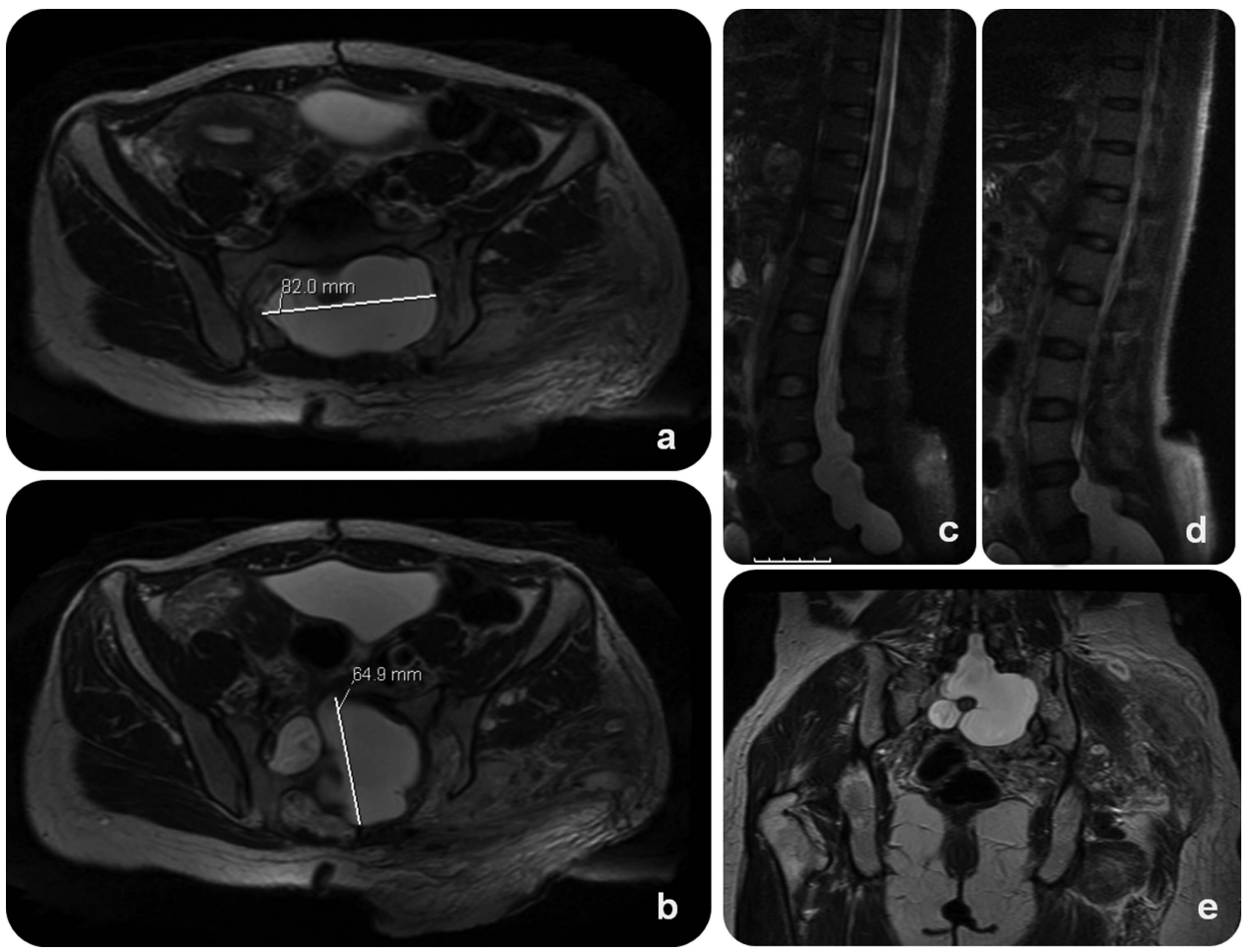

Figure 2 MRI in T2-weighted images: axial scans (A and B) showing a $65 \times 82 \mathrm{~mm}$ hyperintense mass, suggestive of a multiplex neurofibroma; in sagital ( $C$ and D) and coronal (E) scans, heterogeneous and infiltrative features were more evident, leading to the hypothesis of a malignant transformation.

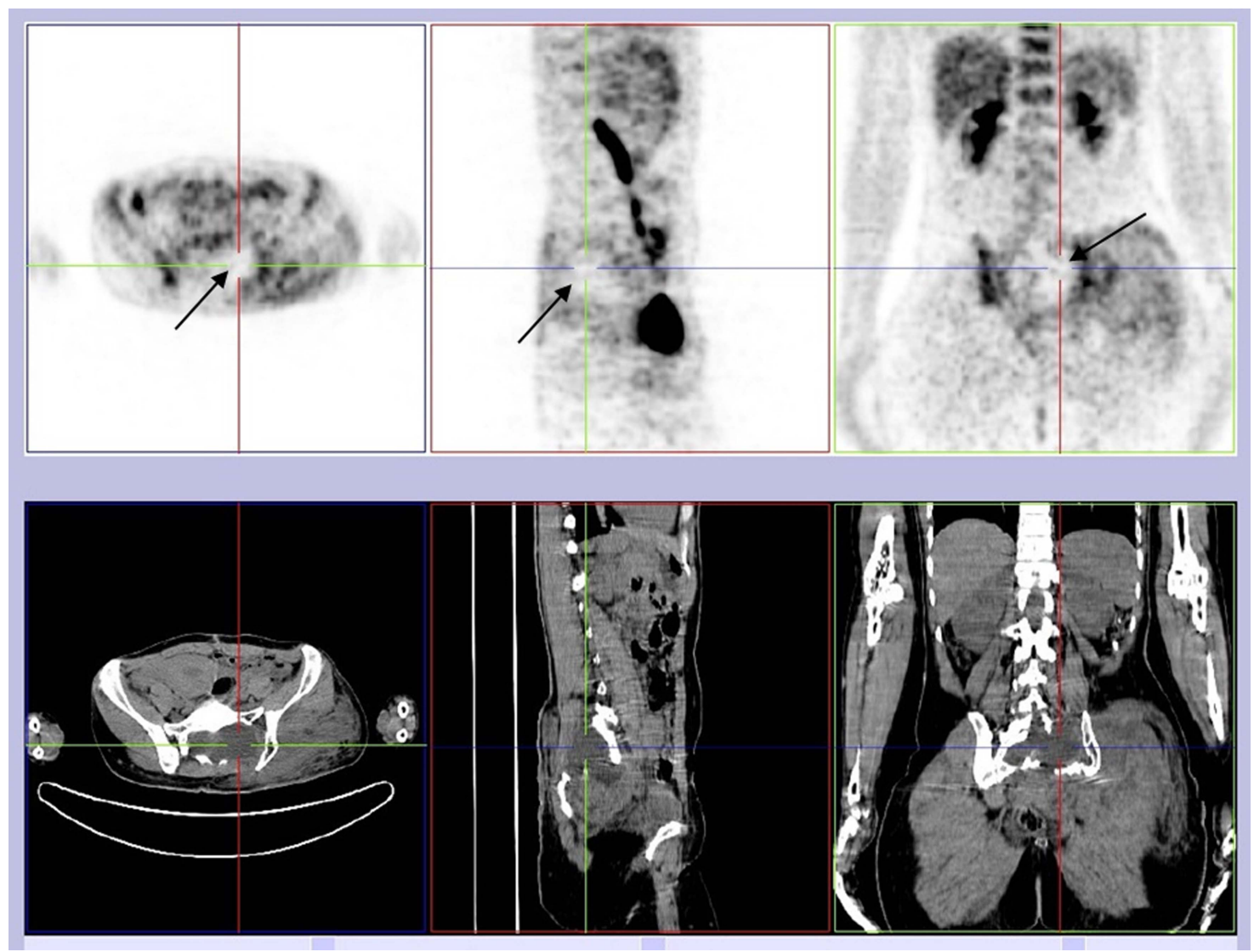

Figure 3 The F-18 fluorodeoxyglucose-positron emission tomography scan images showing a poor tracer uptake by the lumbar mass (signaled by the black arrow), suggesting a benign aetiology such as an internal complex plexiform neurofibroma. 
hormone, vitamin $\mathrm{B}_{12}$ and folate serum levels, serological studies for HIV, hepatitis B and C). The genetic testing for hereditary motor and sensory neuropathy type 1 was not performed.

MRI confirmed a huge and infiltrative mass (figure 2), poorly delimited and located on the left sciatic nerve route, extending from the sacroiliac region to the gluteus muscles (hyperintense lesion in T2); several small nodules could be seen inside, hypointense in T1 and hyperintense in T2 and were suggestive of neurofibromas. After contrast, the mass had heterogeneous uptake and infiltration of the subcutaneous adjacent tissue was seen. Although this lesion could be a plexiform neurofibroma with oedema and muscular denervation, it showed an atypical and aggressive behaviour and a malignant transformation into a MPNST could not be excluded. Considering the high surgical risk of the location, the biopsy was delayed and a positron emission tomographic scan was performed. In this examination, the mass had a poor uptake of F-18 fluorodeoxyglucose (figure 3), indicating a lower level of inflammation. Since this behaviour is not typical of proliferating aggressive tumours (which shows an enhanced tracer uptake), a malignant transformation was unlikely. Thus, a benign aetiology was considered in the form of an internal complex plexiform neurofibroma.

The patient was maintained under medical management, with pain control and radiological surveillance. Two years later this complex plexiform neurofibroma remains stable, without significant growth and without neuropathy progression, as described in the literature ${ }^{1}$ but a continuous and close surveillance remains absolutely necessary.

\section{Learning points}

- Plexiform neurofibromas (PNs) are benign tumours arising from Schawn cells, but they can be locally aggressive (in 64\%), causing pain and functional impairment. ${ }^{12}$

- Internal PNs have more risk of malignant transformation into malignant peripheral nerve sheath tumour (signalled by a rapid increase in tumour size, worsening pain and new neurological deficits) and can be detected only by images studies. ${ }^{2} 3$

- The management of these complex lesions is unclear; the surgical approach is particularly difficult because they are extensively infiltrative, highly vascularised and tend to recur. $^{2} 3$

\section{Competing interests None.}

Patient consent Obtained.

Provenance and peer review Not commissioned; externally peer reviewed.

\section{REFERENCES}

1 Drouet A, Wolkenstein P, Lefaucheur JP, et al. Neurofibromatosis 1-associated neuropathies: a reappraisal. Brain 2004;127:1993-2009.

2 Nguyen R, Dombi E, Widemann BC, et al. Growth dynamics of plexiform neurofibromas: a retrospective cohort study of 201 patients with neurofibromatosis 1 . Orphanet J Rare Dis 2012;7:75.

3 Leroy K, Dumas V, Martin-Garcia N, et al. Malignant peripheral nerve sheath tumors associated with neurofibromatosis type 1. Arch Dermatol 2001;137:908-13.

\footnotetext{
Copyright 2013 BMJ Publishing Group. All rights reserved. For permission to reuse any of this content visit http://group.bmj.com/group/rights-licensing/permissions.

BMJ Case Report Fellows may re-use this article for personal use and teaching without any further permission.

Become a Fellow of BMJ Case Reports today and you can:

- Submit as many cases as you like

- Enjoy fast sympathetic peer review and rapid publication of accepted articles

- Access all the published articles

- Re-use any of the published material for personal use and teaching without further permission

For information on Institutional Fellowships contact consortiasales@bmjgroup.com

Visit casereports.bmj.com for more articles like this and to become a Fellow
} 